\title{
Estimation of Stresses in a Dry Sand Layer Tested on Shaking Table
}

\author{
Andrzej Sawicki*, Marek Kulczykowski*, Robert Jankowski** \\ * Institute of Hydro-Engineering, Polish Academy of Sciences, ul. Kościerska 7, 80-328 Gdańsk, Poland, \\ e-mails: as@ibwpan.gda.pl, marek@ibwpan.gda.pl (corresponding author), \\ ** Gdańsk University of Technology, Narutowicza 11/12, 80-233 Gdańsk, Poland, \\ e-mail: robert.jankowski@wilis.pg.gda.pl
}

(Received September 21, 2011; revised March 29, 2012)

\begin{abstract}
Theoretical analysis of shaking table experiments, simulating earthquake response of a dry sand layer, is presented. The aim of such experiments is to study seismic-induced compaction of soil and resulting settlements. In order to determine the soil compaction, the cyclic stresses and strains should be calculated first. These stresses are caused by the cyclic horizontal acceleration at the base of soil layer, so it is important to determine the stress field as function of the base acceleration. It is particularly important for a proper interpretation of shaking table tests, where the base acceleration is controlled but the stresses are hard to measure, and they can only be deduced. Preliminary experiments have shown that small accelerations do not lead to essential settlements, whilst large accelerations cause some phenomena typical for limit states, including a visible appearance of slip lines. All these problems should be well understood for rational planning of experiments. The analysis of these problems is presented in this paper. First, some heuristic considerations about the dynamics of experimental system are presented. Then, the analysis of boundary conditions, expressed as resultants of respective stresses is shown. A particular form of boundary conditions has been chosen, which satisfies the macroscopic boundary conditions and the equilibrium equations. Then, some considerations are presented in order to obtain statically admissible stress field, which does not exceed the Coulomb-Mohr yield conditions. Such an approach leads to determination of the limit base accelerations, which do not cause the plastic state in soil. It was shown that larger accelerations lead to increase of the lateral stresses, and the respective method, which may replace complex plasticity analyses, is proposed. It is shown that it is the lateral stress coefficient $K_{0}$ that controls the statically admissible stress field during the shaking table experiments.
\end{abstract}

Key words: granular soils, cyclic loading, shaking table, dynamics

\section{Introduction}

Shaking table experiments are successfully used in earthquake engineering to simulate the seismic behaviour of structures, see Dolce et al (2005), Midorikawa et al (2006), as well as granular soils, see Koga and Matsuo (1990), Prasad et al (2004). It is known that earthquake excitations lead to excess settlements of granular subsoils 
or to their liquefaction, if they are water saturated and work in undrained conditions. Many geotechnical problems are reduced to study a one-dimensional behaviour of a granular column subjected to the cyclic horizontal acceleration at its base, as shown in Fig. 1. This scheme corresponds to a dry granular layer resting on a rigid bedrock, which is subjected to earthquake excitation. Such an excitation induces upwards shear wave propagation in a soil layer, which leads either to its compaction in dry or fully drained conditions or to pore-pressure generation and liquefaction in undrained conditions.

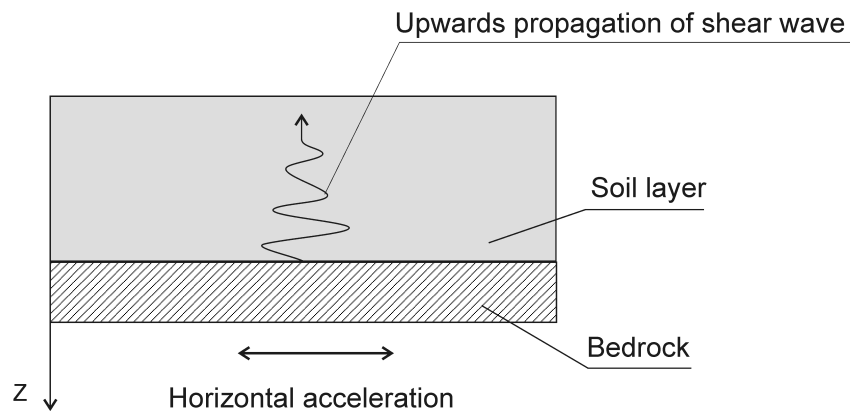

Fig. 1. Upwards shear wave propagation of bedrock shaking - classical geotechnical scheme

An exact simulation of the behaviour of system presented in Fig. 1 on the shaking table is not obvious and straightforward, as it will be explained below. Fig. 2 shows a scheme of shaking table located at the Gdańsk University of Technology, which was adapted to conduct dynamic geotechnical investigations. Originally, the setup was designed for dynamic testing of civil engineering structures. It is a unidirectional device, with the moving platform of dimensions $75 \times 60 \mathrm{~cm}$, excited by the PARKER ET50 linear actuator with the stroke of $500 \mathrm{~mm}$, maximum acceleration of $10 \mathrm{~m} / \mathrm{s}^{2}$ and maximum thrust force of $3300 \mathrm{~N}$, cf. Jankowski (2010a, b).

The layer of model "Gdynia" sand was placed in a glass box (aquarium), which was rigidly fixed to the moving platform of shaking table. Then, the assumed horizontal acceleration history of the platform was applied and the behaviour of soil layer observed. Mostly, the simplest sinusoidal acceleration time history was applied:

$$
A=A_{0} \sin \omega t,
$$

where $\omega$ denotes the angular frequency, $t$ is the real time and $A_{0}$ is the amplitude of acceleration.

Preliminary investigations, performed for the sinusoidal acceleration time history, as well as for some other excitations, have shown that the dry soil layer compacts under such loadings, provided that the amplitude of cyclic base acceleration is large enough. The other important fact is that certain localizations of failure, in the form of Rankine edges, have been observed, see Fig. 2. This phenomenon was noticed first by dr J. Mierczyński during the experiments with large amplitudes of acceleration. 


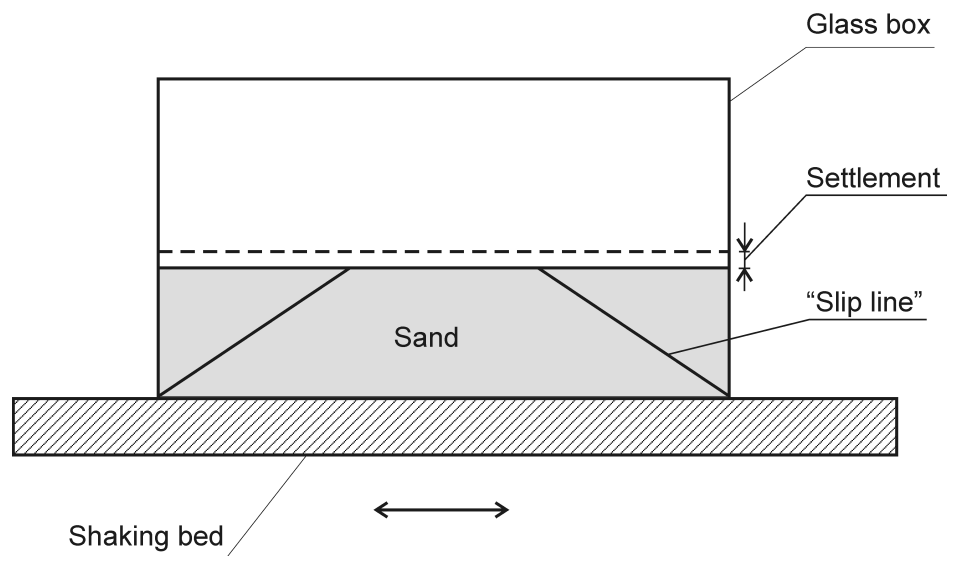

Fig. 2. Scheme of shaking table experiment. Slip lines are caused by large amplitudes of acceleration

The above picture is different from that shown in Fig. 1, as the influence of rigid vertical walls of aquarium is visible in the form of potential slip lines. Such a situation generally does not occur in the case of dynamical scheme presented in Fig. 1, which can be treated as one-dimensional.

The aim of this paper is to estimate the statically admissible stress state in sand tested on the shaking table. First, some heuristic considerations about the dynamics of experimental system are presented. Then, the analysis of boundary conditions, expressed as resultants of respective stresses is shown. A particular form of boundary conditions has been chosen, which satisfies the macroscopic boundary conditions and the equilibrium equations. Then, some considerations are presented in order to obtain statically admissible stress field, which does not exceed the Coulomb-Mohr yield conditions. Such an approach leads to determination of the limit base accelerations, which do not cause the plastic state in soil. It was shown that larger accelerations lead to increase of the lateral stresses, and the respective method, which may replace complex plasticity analyses, is proposed. It is shown that it is the lateral stress coefficient $K_{0}$ that controls the statically admissible stress field during the shaking table experiments.

\section{Heuristic Considerations}

Consider dynamics of a rigid block resting on a shaking platform as shown in Fig. 3. The meaning of $R^{*}$ will be explained later. At present $R^{*}=0$. The weight of a block is $Q$, and the coefficient of friction between the block and shaking bed is $\mu$. Assume the sinusoidal time history of platform's shaking, given by Eq. (1). If the horizontal base acceleration is small enough, the block moves together with the base, i.e. their horizontal displacements, velocities and accelerations are the same. This is the resultant of friction $F$, at the interface between the block and shaking bed, which is the block's 
driving force. It follows from the equilibrium of horizontal forces that $F=m a$, where $a$ denotes the block's acceleration, $m=Q / g$ is the block's mass and $g$ denotes the gravity acceleration. The resultant friction force cannot exceed its maximum value, i.e. $F \leq F_{\max }=\mu Q$, which leads to the following inequality:

$$
|A|<\mu g .
$$

If the condition (2) is satisfied, a common motion of the block and shaking platform takes place. If $|A| \geq \mu g$ a relative movement of the block with respect to accelerating bed takes place, see Sawicki and Chybicki (2005), Sawicki et al (2007).

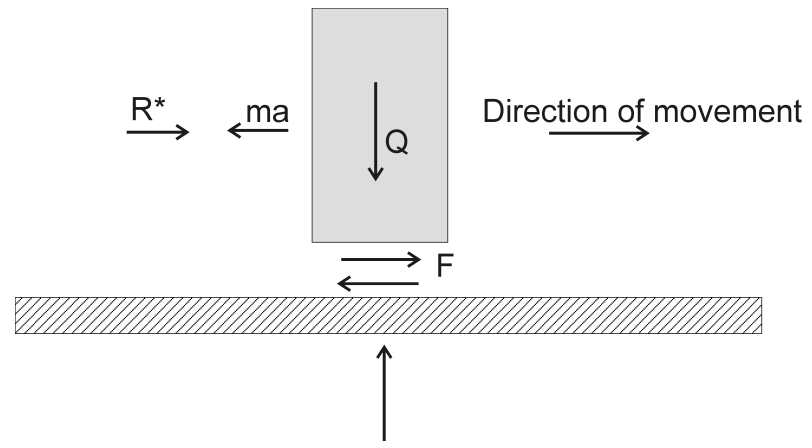

Fig. 3. Forces acting on a rigid block resting on accelerating platform

Consider now a situation, when the block from Fig. 3 cannot move with respect to the bed, because of some horizontal constraints imposed on such a relative motion. Obviously, some reaction $R^{*}$ will appear in these constraints, which is the following:

$$
R^{*}=m a-F=m A-F,
$$

as it was assumed that accelerations of the block and bed should be the same. This reaction disappears when the condition (2) is satisfied again.

The above example can help in better understanding of dynamics of the system shown in Fig. 2. Just imagine that the block shown in Fig. 3 corresponds to sand filling the aquarium in Fig. 2. It is obvious that the sand itself cannot take a rectangular shape, and it should be supported by the vertical walls to retain such a shape. These vertical walls of aquarium impose constraints on potential relative movement of a soil mass with respect to accelerating bed. The soil mass is going to behave like a rigid block, when the condition (2) is not fulfilled, but the vertical walls prevent a relative motion. This means that some reactions between the sand and vertical walls of aquarium are generated. Fig. 2 suggests that these reactions cyclically change, which is an important presumption for analytical studies.

Therefore, an important problem is to determine the walls' reactions, as they would be the boundary conditions for the initial/boundary value problem which 
should be formulated for the soil. The rigid block example, described above, shows that for low accelerations such a dynamic reaction should be zero. The only reaction would be a geostatic state, the same on the right and left hand sides of the aquarium. For larger accelerations, the boundary conditions should change cyclically around this geostatic state, up to the situation when the Coulomb-Mohr yield condition in the soil is reached. After that, the soil behaviour becomes very complex. Fig. 2 suggests that some slip lines develop, and that they correspond to the passive earth pressure according to classical Rankine solutions, which is the other important empirical observation. These experimental observations raise some other questions. For example, what does it mean "small" or "large" acceleration, and how to quantify them?

Subsequent question deals with the geometry of the soil layer. If the ratio of soil's length to its depth is large enough, the Rankine slip lines can be generated. Respective dimensions can be calculated easily, using the methods of classical soil mechanics, e.g. Lambe and Whitman (1969). But in the extreme case of "slim" layer, i.e. when the ratio of soil's length to its depth is small, such classical lines would not appear, and the behaviour of the system becomes extremely complicated. In this paper, the first case of large ratio length/depth will be considered.

\section{Equations for Stresses in Soil}

Assume that the mass of sand retains its rectangular shape as shown in Fig. 2. This mass moves within the rigid system of shaking table (shaking table itself and aquarium), which imposes the basic constraint on such a movement, i.e. the sand should move together with the apparatus. The basic difference between the sand and the shaking table system is that the sand is deformable whilst the shaking table system can be considered as rigid.

Equations of equilibrium for the mass of sand are the following:

$$
\begin{aligned}
& \frac{\partial \sigma_{z}}{\partial z}+\frac{\partial \tau}{\partial x}=\gamma, \\
& \frac{\partial \sigma_{x}}{\partial x}+\frac{\partial \tau}{\partial z}=\rho a,
\end{aligned}
$$

where $\sigma_{x}, \sigma_{z}, \tau$ are components of the stress tensor, $\gamma=\rho g$ is the own weight of soil, $\rho$ denotes the soil density and $a$ is the acceleration of the soil element, which in the case considered is equal to the shaking platform acceleration $A$. The vertical axis of co-ordinates $z$ is directed downwards, and $x$ denotes the horizontal co-ordinate. The upper surface of soil layer corresponds to $z=0$. This system of co-ordinates moves together with the soil. The soil mechanics sign convention is applied when compression is positive.

The stress state in the soil should not exceed the Coulomb-Mohr yield condition:

$$
f=\left(\sigma_{z}-\sigma_{x}\right)^{2}-\left(\sigma_{z}+\sigma_{x}\right)^{2} \sin ^{2} \varphi+4 \tau^{2} \leq 0,
$$


where $\varphi$ denotes the angle of internal friction.

Before shaking begins, the geostatic stress state in the soil exists, i.e. $\sigma_{z}=\gamma z, \sigma_{x}=$ $K_{0} \sigma_{z}$, where $K_{0}$ is a coefficient of lateral pressure.

Note that the relations (4)-(6) are not sufficient for determination of the stress state in the soil, as we have just two differential equations (4) and (5), and an algebraic constraint for stresses (6). Only in the soil's limit state, i.e. when $f=0$, one obtains three equations for determination of three unknown functions. The theory of limit states is well elaborated in soil mechanics, see Chen (1975) or Derski et al (1988). In the case of pre-failure behaviour, i.e. when $f<0$, Eqs. (4) and (5) are insufficient to determine the stress state, so additional relations should be formulated. Another important issue is the problem of boundary conditions, which are dynamic in the case analyzed and unknown at present.

\section{Resultant Boundary Conditions}

\subsection{General Equilibrium}

Heuristic considerations presented in Section 2 indicate that respective reactions of the aquarium should be determined first. Let us consider a macroscopic situation, when we deal with the resultants of respective reactions only, which are imposed on the rectangular shape of the soil mass. The distribution of boundary stresses, corresponding to these resultant reactions, is another important issue. Recall that we would like to satisfy the basic requirement of general mechanics, which states that all the external forces acting on the body should satisfy respective equilibrium equations. Such external forces, in the form of resultant reactions, should satisfy this requirement first. A general situation is shown in Fig. 4.

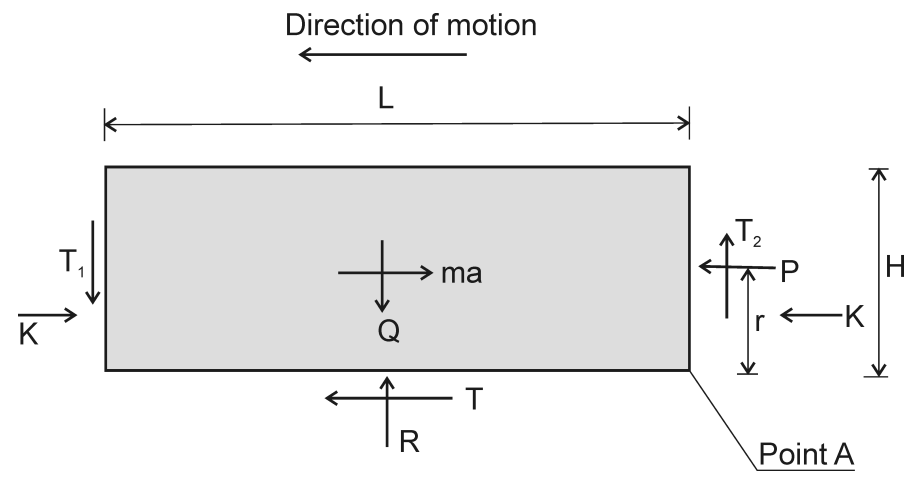

Fig. 4. General equilibrium of the soil mass

$Q=\gamma H L$ is the own weight of the soil mass per $1 \mathrm{~m}$ of depth, perpendicular to the $x z$ plane, $R$ is the vertical reaction of the platform, $m a$ denotes the inertial force, $T$ is the driving force which moves the soil mass according to the platform 
movement, $T_{1}$ and $T_{2}$ are the resultants of shearing stresses acting on the vertical walls of aquarium, and $P$ is the resultant of horizontal reactions of vertical walls of the aquarium, $r$ denoting a position of $P$. Altogether we have the following six unknowns: $R, P, r, T, T_{1}, T_{2}$, whilst there are only three equilibrium equations to determine these reactions, according to classical structural mechanics. $K$ denotes the resultants from geostatic reactions.

In order to make this problem statically determinated task, some additional equations are necessary. For example, if we assume that $T_{1}=T_{2}$, the number of unknowns will be reduced, and also we can determine the platform's reaction $R=Q$. This means that there are four unknowns, namely $P, r, T, T_{1}$, but only two equations remain for their determination. The other additional equations can be chosen as follows: $T=\mu Q$ (valid when $A>\mu g$ ) and $r=H / 3$. In this case:

$$
\begin{gathered}
P=\rho H L(A-\mu g), \\
T_{1}=\frac{1}{3} \rho H^{2}\left(\frac{1}{2} A+\mu g\right),
\end{gathered}
$$

where $\rho=\gamma / g$ denotes the soil density.

\subsection{Particular Case $A<\mu g$}

In this case, according to analogy with the rigid block motion, the resultant of friction forces at the base $T<\mu Q$, so the condition for relative motion is not satisfied and $T$ becomes unknown. Assume particular boundary conditions, i.e. $T_{1}=0, T_{2}=$ $T H / L, r=H / 3$. Therefore, there are three unknowns $(P, R, T)$ and three equilibrium equations, so the problem is statically determinate. From the equilibrium of vertical and horizontal forces, and from the condition that a sum of moments with respect to point $\mathrm{A}$ is zero, one obtains the following equations:

$$
\begin{gathered}
Q-R-\frac{T H}{L}=0, \\
m A-P-T=0, \\
\frac{m A H}{2}-\frac{Q L}{2}+\frac{R L}{2}-\frac{P H}{3}=0 .
\end{gathered}
$$

The solution is as follows:

$$
P=0, \quad T=m A, \quad R=\gamma H\left(L-\frac{A H}{g}\right) .
$$

It is an interesting result, as $P=0$, which is consistent with analogy to the rigid block motion. 


\subsection{Some Numerical Estimates}

The following data correspond to experimental results: $L=50 \mathrm{~cm}, H=16 \mathrm{~cm}$, frequency $f=1 \mathrm{~Hz}$ and $A_{0}=0.2 g$ (cf. Eq. (1)). For these data $R=0.936 Q, T=0.2 Q$ and $T_{2}=0.064 Q$. Note that these values correspond to the maximum (peak) acceleration. Recall that there are also the resultants of horizontal stresses $K$ which follow from the assumed geostatic state.

The above data suggest that a reasonable approximation of the resultant boundary conditions would be if we assume: $R=Q$ and $T_{1}=0$. Such a simplification enables determination of stresses in the soil in a simple way.

\section{Estimation of Stresses in the Soil within a Single Cycle of Shaking}

\subsection{Simple Stress Field}

Assume that the shearing stresses at the base of soil layer are uniformly distributed:

$$
\tau(z=H)=\tau_{0}=\frac{T}{L}=\rho A H .
$$

Also assume the geostatic state for normal stresses, i.e.

$$
\sigma_{z}=\gamma z, \quad \sigma_{x}=K_{0} \gamma z
$$

Note that Eq. (4) is satisfied if one assumes that the shear stress does not depend on $x$ and is only a function of $z$. Eq. (5) reduces to the following relation:

$$
\frac{\partial \tau}{\partial z}=\rho A,
$$

from which one obtains:

$$
\tau=\rho A z \text {. }
$$

Note that Eq. (16) satisfies the boundary condition (13). It should also be noted that the shape of relation (16) is similar to the shear stress distribution obtained assuming the mechanism of shear wave propagation shown in Fig. 1, cf. Sawicki and Świdziński (1989). The simplified solution given by Eqs. (14) and (16) may not be very accurate in the vicinity of vertical walls of the aquarium, but if $L>>H$ it becomes an exact solution for one-dimensional problem of the shear wave propagation. Recall again that the above result is valid when $A<\mu g$.

\subsection{Statically Admissible Stresses}

The stress field given by Eqs. (14) and (16) should be statically admissible, which means that it should satisfy the equilibrium equations with respective boundary conditions and should not exceed the Coulomb-Mohr yield condition, cf. inequality (6). 
The first requirement is already satisfied and we have to check the second one. Substitution of Eqs. (14) and (16) into (6) leads to the following inequality:

$$
\left(\frac{A}{g}\right)^{2} \leq \frac{1}{4}\left[\left(1+K_{0}\right)^{2} \sin ^{2} \varphi-\left(1-K_{0}\right)^{2}\right],
$$

from which the acceleration causing the plastic state in soil can be determined.

As an example, consider the case when $K_{0}=0.5$ and $\varphi=30^{\circ}$. Substitution of these data into the RHS of inequality (17) gives 0.078 . It means that the acceleration $A / g=\sqrt{0.078}=0.28$ would initiate the plastic state in soil. The above mentioned amplitude of acceleration $A_{0}=0.2 g$ will not lead to the plastic state of soil.

The basic question is what would happen if the acceleration of the bed increases above admissible level causing the plastic state in soil. A classical procedure would require the analysis of either rigid-plastic or elasto-plastic problem in which the re-grouping of the stress state takes place. In this paper such a procedure will be shortened, as we assume that this is the $K_{0}$ parameter that controls this requirement. It should be determined from relation (17) where the inequality should be replaced by equality. This increase of $K_{0}$ can be explained on the basis of some physical considerations, namely by compaction of sand caused by cyclic shearing which, in turn, changes lateral stresses.

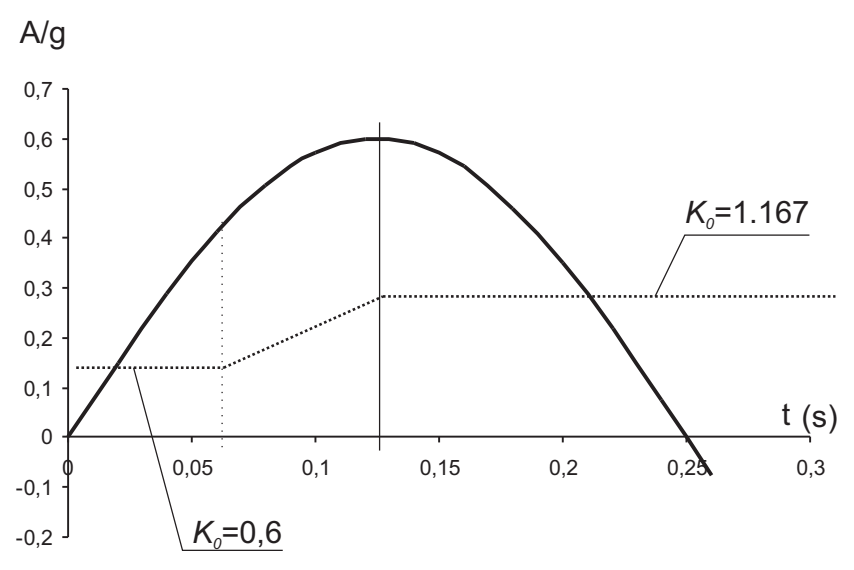

Fig. 5. The change of coefficient $K_{0}$ when the platform acceleration exceeds admissible level. The first half of a cycle. The soil adapts to excess acceleration by increasing lateral stresses

Fig. 5 illustrates the above mechanism. It shows the first half of a sinusoidal cycle, when the amplitude of acceleration is $A_{0}=0.6 \mathrm{~g}$ and the frequency is $f=2 \mathrm{~Hz}(\omega=$ $\left.12.57 \mathrm{~s}^{-1}\right)$. Assuming $\varphi=34^{\circ}$ and initial value of the coefficient of earth pressure at rest $K_{0}=0.6$, which correspond to tested "Gdynia" sand, the maximum acceleration at which the Coulomb-Mohr yield condition is reached is $0.4 \mathrm{~g}$. If the acceleration exceeds this level, the relation (6) would be violated, for the assumed data, which 
cannot be accepted from the physical point of view. Therefore the lateral stress should increase in order to keep the stress state on the limit surface. This means that this is the coefficient $K_{0}$ which should increase as the vertical stresses do not change. The change of this coefficient is calculated from relation (17):

$$
K_{0}=\frac{1}{1-M}[1+M-\sqrt{4 M-(1-M) N}],
$$

where $M=\sin ^{2} \varphi$ and $N=4\left(A_{0} / g\right)^{2} \sin ^{2} \omega t$.

For the assumed data, Eq. (18) takes the following form:

$$
K_{0}=1.455\left(1.3127-\sqrt{1.251-0.99(\sin 12.57 t)^{2}}\right),
$$

where the argument of sinus function is expressed in radians. Eq. (18) is valid for the time interval $\{0.058-0.125 \mathrm{~s}\}$. At $t=0.058 \mathrm{~s}$, which corresponds to the initial data and the acceleration of $0.4 \mathrm{~g}$, the Coulomb-Mohr yield condition is reached for the first time. Then, the base acceleration increases above this level, reaching its peak value of $0.6 \mathrm{~g}$ at $t=0.125 \mathrm{~s}$. During this interval, the coefficient $K_{0}$ almost linearly increases, according to Eq. (18), and finally reaches its maximum value of 1.167. Then, this coefficient remains constant, provided that the maximum acceleration does not exceed the level of $0.6 \mathrm{~g}$. For acceleration amplitudes larger than $0.6 \mathrm{~g}$, the above procedure should be repeated.

Note that Eq. (18) has been derived from relation (17) as one of roots of respective quadratic equation. For some combination of data, a square root appearing in this equation may take negative values. In a special case considered, it is equal to zero for $A_{0}=0.675 \mathrm{~g}$, which formally determines a range of application of the approach proposed. On the other hand, the shaking table experiments performed for such large values of acceleration display an unusual behaviour of sand layer, difficult to describe using classical methods as those presented in this paper. Large cyclic vertical displacements, particularly in the vicinity of vertical walls, are observed. The sand behaviour does not resemble reaction of a solid-like material, but rather a complex motion of a kind of "granular fluid". We do not study such a mechanism in the present paper. Recall that the aim of this paper is to determine statically admissible stress field which could be used in subsequent determination of sand compaction.

\subsection{Numerical Estimation of Stresses}

It is important in experimental work to assess expected stresses and other quantities for rational designing of these experiments and even for economic reasons such as, for example, purchase of stress gauges. Note that Prasad et al (2004) have designed their simple shaking table just for economic reasons! Our experiments were performed on "Gdynia" sand, the own weight of which is $\gamma=16.35 \mathrm{kN} / \mathrm{m}^{3}$. Therefore, the maximum vertical stress is the following: $\sigma_{z}=\gamma H=2.616 \times 10^{3} \mathrm{~N} / \mathrm{m}^{2} \approx 0.03 \times 10^{5}$ 
$\mathrm{N} / \mathrm{m}^{2}$. This is a very small stress, almost equal to accuracy of commercially available gauges. A similar order of magnitude can be expected for pore pressures, generated in saturated sand subjected to cyclic loadings.

Note that the shaking table experiments are specific as they are performed at $1 \mathrm{~g}$ conditions, and in a small geometrical scale. In such a situation, no scaling laws can be applied so the system should be treated as a small prototype. This is because the properties of sand as, for example, their various moduli strongly depend on the mean effective stress. In the shaking table experiments we deal with small stresses, which could hardly be measured by available gauges. Therefore, a rational theoretical analysis is of a great help.

\section{Discussion and Conclusions}

The main results reported in this paper can be summarized as follows:

1. A simple analysis of the problem of stress estimation during the shaking table experiments was presented. This analysis is based on classical methods of general and continuum mechanics, as well as on the experimental results.

2. The requirements of general and continuum mechanics have been collated, and the statically admissible stress field has been determined. This stress field satisfies equilibrium equations and respective boundary conditions. Practically useful solution has been obtained by certain simplification of the stress boundary conditions at vertical wall of aquarium. This means that this solution is slightly violated in this region, so it is a good approximation for large ratios $L / H$. For slim layers, i.e. when the ratio $L / H$ is small, the solution proposed does not apply.

3. An interesting feature of the solution obtained is that the cyclic shear stresses are the same as those obtained for the problem of upwards shear wave propagation in a soil stratum subjected to the cyclic base acceleration.

4. The above solution depends on the magnitude of platform's acceleration. A method for determination of the limit acceleration, which does not cause the plastic state in the soil, was proposed. For larger accelerations, the other method was proposed. It was shown that excess accelerations induce some additional lateral stresses in the soil, such as the Coulomb-Mohr yield condition is not exceeded. It was shown that in order to satisfy the above requirements, the coefficient of lateral earth pressure should increase. A method has been proposed that enables the determination of above mentioned coefficient, and subsequent behaviour of the soil layer. Respective stresses have been estimated.

5. The main result of this paper was to show that excess accelerations induce additional lateral stresses in the soil, and to propose respective method of determination of these shaking-induced stresses. 


\section{Acknowledgement}

Research presented in this paper was supported by the Polish Ministry of Science and Higher Education (project N N506 072938).

\section{References}

Chen W. F. (1975) Limit Analysis and Soil Plasticity, Elsevier, Amsterdam.

Derski W., Izbicki R., Kisiel I., Mróz Z. (1988) Rock and Soil Mechanics, PWN Warsaw \& Elsevier, Amsterdam/Oxford/New York/Tokyo.

Dolce M., Cardone D., Ponzo F. C., Valente C. (2005) Shaking table tests on reinforced concrete frames without and with passive control systems, Earthquake Engineering and Structural Dynamics, 34, 1687-1717, doi: 10.1002/eqe.501.

Jankowski R. (2010a) Experimental study on earthquake-induced pounding between structural elements made of different building materials, Earthquake Engineering and Structural Dynamics, 39, 343-354. doi: 10.1002/eqe.941.

Jankowski R. (2010b) Shaking table experimental study on diagnosis of damage and its evaluation in steel structure, Key Engineering Materials, 417-418: 157-160, doi: 10.428/www.scientific.net/KEM. 417-418.157.

Koga Y., Matsuo O. (1990) Shaking table tests of embankments resting on liquefiable sandy ground, Soils and Foundations, 30, 162-174.

Lambe T. W., Whitman R. V. (1969) Soil Mechanics, J. Wiley \& Sons, N. York/London/Sydney/ Toronto, Polish translation (1977) Arkady, Warsaw.

Midorikawa M., Azuhata T., Ishihara T., Wada A. (2006) Shaking table tests on seismic response of steel braced frames with column uplift, Earthquake Engineering and Structural Dynamics, 35, 1767-1785, doi: 10.1002/eqe.603.

Prasad S. K., Towhata I., Chandradhara G. P., Nanjundaswamy P. (2004) Shaking table tests in earthquake geotechnical engineering, Current Science, 87 (10), 1398-1404.

Sawicki A., Chybicki W. (2005) Horizontal motion of a rigid block resting on accelerating subsoil, Archives of Hydro-Engineering \& Environmental Mechanics, 52 (2), 147-160.

Sawicki A., Chybicki W., Kulczykowski M. (2007) Influence of vertical ground motion on seismic-induced displacements of gravity structures, Computers \& Geotechnics, 34, 485-497, doi:10.1016/j.compgeo.2006.12.002.

Sawicki A., Świdziński W. (1989) Mechanics of a sandy subsoil subjected to cyclic loadings. Int. Jnl for Numerical and Analytical Methods in Geomechanics, 13, 511-529, doi: 10.1002/nag.1610130505. 